\title{
Metallic Microswimmers Driven up the Wall by Gravity
}

\author{
Quentin Brosseau ${ }^{1}$, Florencio Balboa Usabiaga ${ }^{2}$, Enkeleida Lushi ${ }^{3}$, Yang $\mathrm{Wu}^{4}$, \\ Leif Ristroph $^{1}$, Michael D. Ward ${ }^{4}$, Michael J. Shelley ${ }^{1,2}$ and Jun Zhang ${ }^{1,5,6}$ \\ 1 Applied Mathematics Laboratory, Courant Institute, New York University, NY NY 10012, USA, \\ 2 Flatiron Institute, Simons Foundation, NY NY 10010, USA \\ 3 Dept. of Math. Sciences, New Jersey Institute of Technology, Newark NJ 07102, USA \\ 4 Dept. of Chemistry, New York University, NY NY 10012, USA \\ 5 Dept. of Physics, New York University, NY NY 10003, USA \\ 6 NYU-ECNU Physics and Mathematics Research Institutes, \\ New York University Shanghai, Shanghai 200062, China
}

\begin{abstract}
Experiments on autophoretic bimetallic nanorods propelling within a fuel of hydrogen peroxide show that tail-heavy swimmers preferentially orient upwards and ascend along inclined planes. We show that such gravitaxis is strongly facilitated by interactions with solid boundaries, allowing even ultraheavy microswimmers to climb nearly vertical surfaces. Theory and simulations show that the buoyancy or gravitational torque that tends to align the rods is reinforced by a fore-aft drag asymmetry induced by hydrodynamic interactions with the wall.
\end{abstract}

PACS numbers:

As part of their survival, many microorganisms, such as the algae C. reinhardtii, E. gracilis, or Paramecia, need to swim up against gravity. Such behavior is known as gravitaxis. These swimmers, when pulled by gravity, align vertically due to a fore-aft drag asymmetry along their bodies that generates a hydrodynamic torque. [1-6 Inhomogeneous density distributions within their bodies can also lead to buoyancy torques and vertical alignment. 7 Once oriented vertically their propulsion allows vertical migration. When in a group, these torques contribute to the emergence of colonial bioconvective patterns and to the stratification of swimmers in the bulk. 8611] Near confining walls, the dynamics of any swimmer is expected to change due not only to gravity but also to hydrodynamic interactions with boundaries. 12, 13 Indeed, many microbes inhabit wet soils and other porous media where sloped boundaries are omnipresent. 14, 15 A natural question is whether such walls will suppress or enhance gravitaxis.

The design of artificial microswimmers can incorporate the working principles underlying organismal gravitaxis to drive, direct, and optimize the motion of self-propelled colloids. 16, 17] For example, spherical polystyrene beads coated with a heavy metallic cap on its trailing pole and fueled by hydrogen peroxide swim up in the bulk. [18, 19. Swimmer shape also affects trajectory, as demonstrated for L-shaped autophoretic colloids (powered by light) swimming on an inclined plane, wherein asymmetric propulsion-to-drag distribution allowed steady upslope movement plus curved motions and sedimentation. [20]

We describe the gravitactic behavior of active bimetallic rods, combining experiments, theory and simulations to demonstrate that these heavy nanomotors can swim up inclined walls, even very steep ones; see Fig. 1. Their behavior resembles some aspects of organismal gravitaxis, as these nanomotors are tail heavy such that density inhomogeneity contributes to an upright orientation
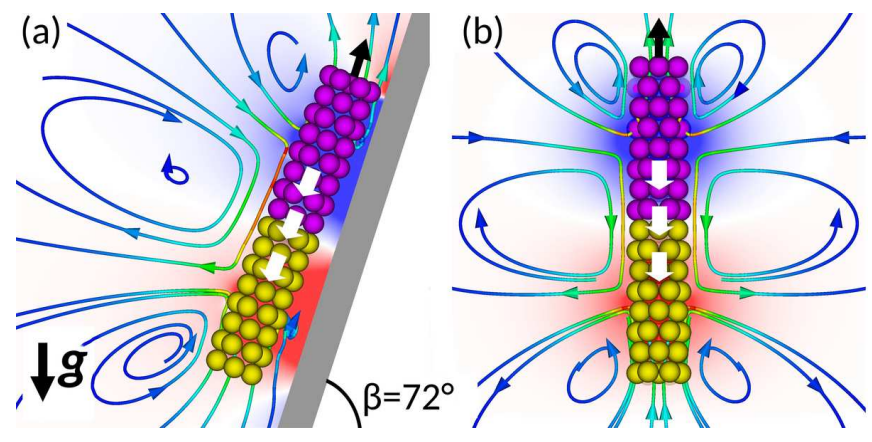

FIG. 1: Climbing rod and flow fields. Computed flow streamlines and regions of high/low (red/blue background) pressure from a simulation of a gold-rhodium rod climbing a steep wall: views from the side (a) and the front of the wall (b). In our model, the reduction and oxidation of $\mathrm{H}_{2} \mathrm{O}_{2}$ on the metallic segments generate an active slip layer (white arrows) near the bimetallic junction, propelling the rod. Notice that the rod has a dynamically determined head-down tilt with respect to the wall.

of the rods. Direct real-time observation reveals that rods of homogeneous density sediment, i.e. do not climb, along the wall. Surprisingly, however, these rods are subject to a gravitactic bias that slows their sedimenting speeds. Our theoretical analysis and simulations demonstrate that the latter result can be explained by an effective fore-aft asymmetry in the hydrodynamic interaction between the rod and the nearby wall. This additional hydrodynamic effect enhances the gravitactic behavior of rods with density inhomogeneity.

\section{EXPERIMENTAL SETUP AND RESULTS}

The bimetallic swimmers used herein were $2.5 \mu \mathrm{m}$ long gold-rhodium $(\mathrm{Au}-\mathrm{Rh})$ or $2.0 \mu \mathrm{m}$ gold-platinum 

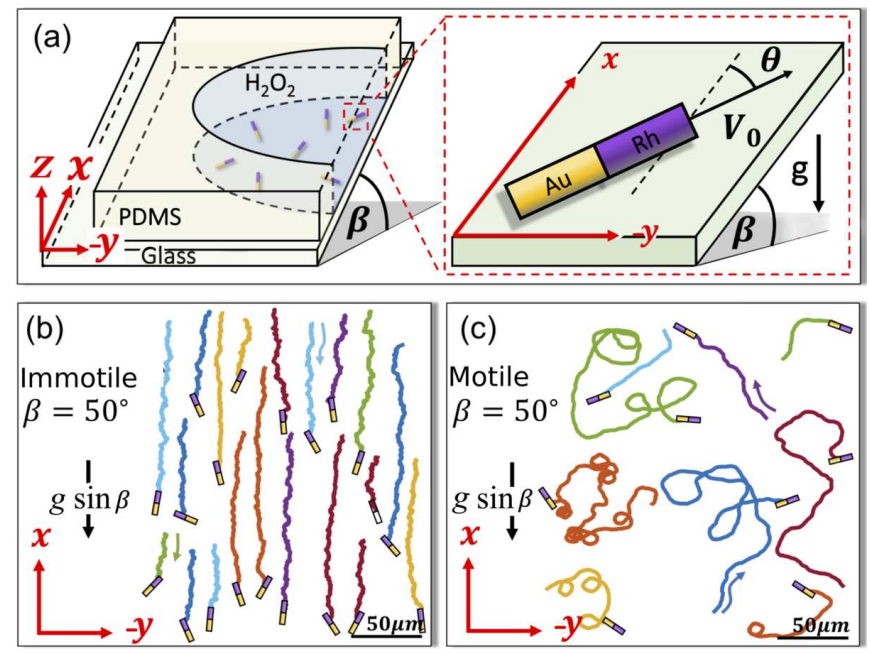

FIG. 2: Au-Rh bimetallic rods moving on an inclined wall. (a) Cut-away view of our experimental setup. The rods were enclosed in a chamber containing $\mathrm{H}_{2} \mathrm{O}_{2}$ solution. The inclination angle $\beta$ is controlled by a super-structure encasing the chamber and optical microscope (not shown). (b) Trajectories acquired over 2 minutes of recording for $\mathrm{Au}-\mathrm{Rh}$ rods on a surface inclined $50^{\circ}$ for immotile rods (size exaggerated) when $\mathrm{H}_{2} \mathrm{O}_{2}$ is absent. (c) Motile rods (with $30 \% \mathrm{H}_{2} \mathrm{O}_{2}$ ) were seen to make random but overall upward motion against gravity, an effect more evident from statistical analysis.

(Au-Pt) rods having diameter $d \approx 0.3 \mu \mathrm{m}$. The rods were synthesized by electrodeposition in anodized aluminum oxide templates according to a previously reported protocol. 21, 22, The metallic segments were either length-symmetric (1:1) Au:Rh or Au:Pt rods, or length-asymmetric (3:1) Au:Pt with long-gold and shortplatinum segments. More details on the rod synthesis is provided in the Supplementary Material. 23.

These rods self-propel when submersed in aqueous hydrogen peroxide $\left(\mathrm{H}_{2} \mathrm{O}_{2}\right)$ solutions as fuel 24. The fuel reduction/oxidation occurs on the $\mathrm{Au} / \mathrm{Pt}$ or $\mathrm{Au} / \mathrm{Rh}$ segments, creating an uneven charge distribution along the rod. The resulting electric field induces ionic migration in the rods' diffuse layer, creating a "slip layer" of fluid that envelops the rod and is likely most pronounced at the junction between the two metals 25. This fluid displacement, due to momentum conservation, results in rod movement in the opposite direction, with the rhodium or platinum segment leading the motion. [26, 27] The geometrically symmetric Au-Rh rods have a density asymmetry of ratio roughly $3: 2$ between the two segments, as $\rho_{\mathrm{Au}}=19.32 \mathrm{~g} / \mathrm{cm}^{3}$ and $\rho_{\mathrm{Rh}}=12.41 \mathrm{~g} / \mathrm{cm}^{3}$. Consequently, the rod Center of Mass (CoM) sits rearwards, resulting in a tail-heavy rod. In contrast, platinum is only slightly denser than gold, $\rho_{\mathrm{Pt}}=21.45 \mathrm{~g} / \mathrm{cm}^{3}$, such that the density of Au-Pt rods is nearly balanced. The fluid density is typically $\rho_{\mathrm{f}} \approx 1.1 \mathrm{~g} / \mathrm{cm}^{3}$, depending on the amount of $\mathrm{H}_{2} \mathrm{O}_{2}$ added to water.

We used a Nikon Eclipse 80i microscope mounted on a custom-made tilting structure that permits adjustment

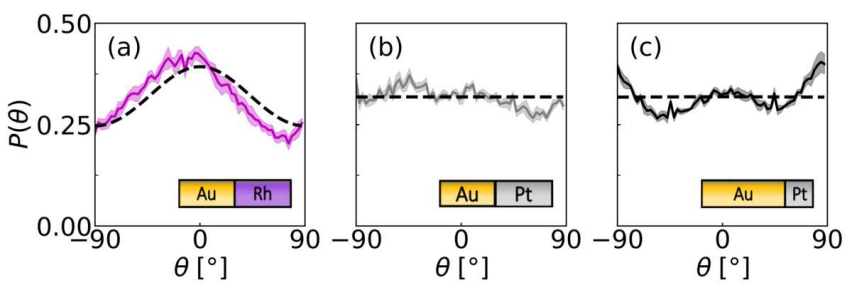

FIG. 3: Orientations of sedimenting immotile rods on inclined walls. Without $\mathrm{H}_{2} \mathrm{O}_{2}$, rods sediment, the distribution of their angles with the $x$-axis, $P(\theta)$, along a wall with inclination $\beta=70^{\circ}$ are shown for (a) Au-Rh tail-heavy rods and $\mathrm{Au}-\mathrm{Pt}$ density-even rods (b)symmetric and (c) asymmetric with long-gold segment. Continuous lines with confidence interval (one std. error) experimental results; dashed lines fit to Eq. (6).

to prescribed inclinations from horizontal to vertical (tilt angle $\left.\beta \in\left[0^{\circ}, 90^{\circ}\right]\right)$. The experimental chamber was mounted on the microscope's stage and positioned to ensure a fixed alignment with the optics. The chamber was a circular well with volume $\sim 1 \mathrm{~cm}^{3}$, cut from a $0.5 \mathrm{~cm}$ thick PDMS slab and mounted on a glass slide, as illustrated in Fig. 22. This chamber was filled with $\mathrm{H}_{2} \mathrm{O}_{2}$ solution, followed by the addition of the bimetallic rods. The chamber was then capped with a coverslip to ensure an optically flat surface for observation and prevent fluid leakage.

The kinematic characterization of the rods was done with the chamber positioned horizontally $\left(\beta=0^{\circ}\right)$, as the rods sediment to the bottom and move about. Their movements in the focal plane of a 40X objective lens was recorded at a rate of 25 frames/s. Typically, the particle motion was measured for 2 minutes, such as the $\mathrm{H}_{2} \mathrm{O}_{2}$ concentration remained constant, and their trajectories analyzed using the MatLab Image Processing Toolbox and a custom-written software. The characteristic swimming speeds under various $\mathrm{H}_{2} \mathrm{O}_{2}$ concentrations (15\%-30\%) were typically from 3 to $8 \mu \mathrm{m} / \mathrm{s}$. 23]

\section{A. Immotile Rods on an Inclined Wall}

In the absence of $\mathrm{H}_{2} \mathrm{O}_{2}, \mathrm{Au}-\mathrm{Rh}$ and $\mathrm{Au}-\mathrm{Pt}$ rods were immotile. Since gravitational force dominates over thermal forces, the rods, unsurprisingly, slid down in rectilinear trajectories (Fig. 2 2b). The distribution of the angle between the rod axis and $\boldsymbol{x}, P(\theta)$, has a maximum at the vertical direction, $\theta=0$, for tail-heavy $\mathrm{Au}-\mathrm{Rh}$ rods (Fig. 3a) and is rather flat for both types of densitybalanced Au-Pt rods (Fig. 3b, c). In the absence of reduction/oxidation reactions (propulsion) the orientation preference can only be linked to the density distribution of the rods. Here, the buoyancy (geometric) center of a $\mathrm{Au}-\mathrm{Rh}$ rod differs from the $\mathrm{CoM}$, giving rise to a torque that reorients the rod. The tail-heavy $\mathrm{Au}-\mathrm{Rh}$ rods sediment with their gold ends leading and long-axis along the gravitational field. 

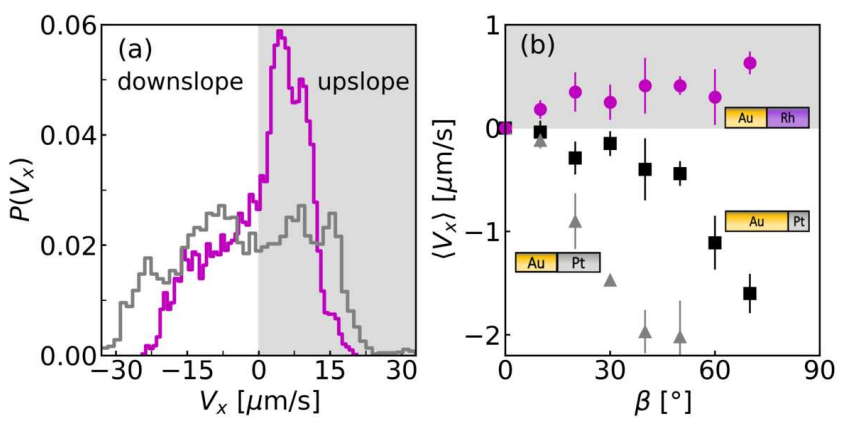

FIG. 4: Velocity of motile rods. (a) Velocity distribution of tail-heavy Au-Rh swimmers — and symmetric densitybalanced, 1:1 Au-Pt swimmers — on a wall with inclination $\beta=70^{\circ}$. Overall, the $\mathrm{Au}-\mathrm{Rh}$ rods swim upslope and perform gravitaxis while $\mathrm{Au}-\mathrm{Pt}$ rods sediment. (b) The average velocity along the $x$-axis $\left\langle V_{x}\right\rangle$ vs. wall inclination $\beta$ for three rod types with comparable swimming speed $V_{0}$ : Au-Rh $\left(\bullet, V_{0}=\right.$ $8.0 \pm 1.0 \mu \mathrm{m} / \mathrm{s})$, symmetric Au-Pt $\left(\Delta, V_{0}=6.1 \pm 0.8 \mu \mathrm{m} / \mathrm{s}\right)$ and long-gold $\mathrm{Au}-\mathrm{Pt}\left(\boldsymbol{\square}, V_{0}=6.5 \pm 0.7 \mu \mathrm{m} / \mathrm{s}\right)$. Au-Rh rods show increasing gravitactic ability with increased inclination, and $\mathrm{Au}-\mathrm{Pt}$ rods sediment at different rates depending on their segmental ratios.

\section{B. Motile Rods}

When submerged in an aqueous solution containing $\mathrm{H}_{2} \mathrm{O}_{2}$ fuel, the rods self-propel along the inclined wall, as illustrated in Fig. 22. Their trajectories become highly nontrivial and exhibit movement up the wall against gravity as well as sideways and downward movements. Their gravitactic behavior is made more evident through statistical analysis of the trajectories. As illustrated in Fig. 4an, the velocity distribution $P\left(V_{x}\right)$ at an inclination $\beta=70^{\circ}$ reveals that tail-heavy $\mathrm{Au}-\mathrm{Rh}$ rods were biased towards upslope swimming. Density-balanced AuPt rods, however, display overall downward movement.

Fig. $4 \mathrm{~b}$ depicts the mean velocity $\left\langle V_{x}\right\rangle$ for different wall inclinations, $\beta$, and for all three swimmer types. Tailheavy $\mathrm{Au}-\mathrm{Rh}$ rods clearly swim upslope. This tendency increases with $\beta$, whereas density-balanced $\mathrm{Au}-\mathrm{Pt}$ rods sediment downslope. Notably, symmetric Au-Pt rods sediment faster downslope than asymmetric 3:1 long-gold $\mathrm{Au}-\mathrm{Pt}$ rods at any inclination $\beta$. The slight gain in mass in the symmetric rod due to the longer Pt segment is not sufficient to explain its faster sedimentation. The next section addresses the role of hydrodynamic interactions between the rods and the wall in the gravitactic response and how it might control the sedimentation speed.

\section{MODELING AND PROVING GRAVITAXIS}

Two methods were used to model gravitaxis. The first is a full hydrodynamic description of the rods and the second is a simplified mechanical model. In the first method, each rod was modeled as a rigid body with an active slip layer centered in the bimetallic junction, see Fig. 1 .
The Stokes equations were solved to determine the surrounding flow and pressure fields in the presence of the wall, and consequently the rod orientation and swimming speed. The Stokes equations were solved with the Rigid Multiblob method. 23, 28, 31] In this method the rod surface is discretized by a set of $N$ blobs with positions $\left(\boldsymbol{r}_{i}-\boldsymbol{q}\right)$ relative to the rod tracking point $\boldsymbol{q}$. The slip condition, Eq. (1), and the balance of force and torque, Eq. (2), form a linear system that is solved for the rod linear and angular velocities, $\boldsymbol{u}$ and $\boldsymbol{\omega}$,

$$
\begin{gathered}
\sum_{j}^{N} \boldsymbol{M}_{i j} \boldsymbol{\lambda}_{j}=\boldsymbol{u}+\boldsymbol{\omega} \times\left(\boldsymbol{r}_{i}-\boldsymbol{q}\right)+\widetilde{\boldsymbol{u}}_{i} \text { for } i=1, \ldots, N \\
\sum_{i}^{N} \boldsymbol{\lambda}_{i}=\boldsymbol{F}, \quad \sum_{i}^{N}\left(\boldsymbol{r}_{i}-\boldsymbol{q}\right) \times \boldsymbol{\lambda}_{i}=\boldsymbol{\tau} .
\end{gathered}
$$

In the above linear system $\boldsymbol{\lambda}_{i}$ is the constraint force acting on the blobs to enforce a rigid motion and $\widetilde{\boldsymbol{u}}_{i}$ is the prescribed active slip on the blobs. The matrix $\boldsymbol{M}$ captures the hydrodynamic interaction between blobs, $\boldsymbol{M}_{i j} \boldsymbol{\lambda}_{j}$ being the flow generated at blob $i$ by the constraint force acting on blob $j$. The matrix $\boldsymbol{M}$ is a regularization of the Green's function of the Stokes equation with the appropriate boundary conditions, i.e. no-slip at the wall. [32, 33] We used the so-called Rotne-Prager approximation with wall corrections which has an analytical expression. 34 The external force and torque, $\boldsymbol{F}$ and $\boldsymbol{\tau}$, acting on the rod included the effect of gravity and the steric interactions with the wall.

In the experiments the active slip was created by the breakdown of $\mathrm{H}_{2} \mathrm{O}_{2}$ near the rod surface. 26, 27, Numerical simulations of the full Stokes-electrochemical equations have shown that the charge gradients created by the fuel reduction/oxidation are larger near the bimetallic junction leading to a stronger slip in that sector. 25] In our numerical modeling instead of solving the complex reduction/oxidation reaction we assumed that a section of the rod surface was covered by an active slip of constant magnitude, $\widetilde{u}_{s}=30 \mu \mathrm{m} / \mathrm{s}$, parallel to the rod axis and centered near the metal-metal junction. 31 Additionally, since the rods worked as a battery with an active anode (Rh/Pt segment) and cathode (Au segment), [26] we assumed that the rate of the redox reaction was controlled by the smaller metal segment. Therefore, we made the length of the active section, $L_{s}$, proportional to the length of the smaller electrode

$$
L_{s}=\left\{\begin{array}{cc}
2 L_{\mathrm{Au}} & \text { if } L_{\mathrm{Au}} \leq L / 4 \\
L / 2 & \text { if } L / 4 \leq L_{\mathrm{Au}} \leq 3 L / 4 \\
L-2 L_{\mathrm{Au}} & \text { if } L_{\mathrm{Au}} \geq 3 L / 4
\end{array}\right.
$$

where $L$ is the length of the rod and $L_{\mathrm{Au}}$ the length of the gold segment.

Our second model aims to understand the observed gravitaxis of $\mathrm{Au}-\mathrm{Rh}$ swimmers and the controllable sedimentation (by different segmental ratios) of Au-Pt swim- 
mers. The swimming rods were observed to remain close to the wall, previous reports have revealed that immotile rods remain parallel to the wall while motile rods swim with a head-down tilt angle $\alpha$ [31, 35]; see Figs. 11 and 5 a inset. The second model assumes that rod trajectories are two-dimensional, in the $x y$-plane parallel to the wall, and it describes the rod configuration by a tracking point (e.g. any fixed point on the $\operatorname{rod}) \boldsymbol{x}(t) \in \mathbb{R}^{2}$ and the rod orientation $\theta(t)$ with respect to the $x$-axis. The rod is now a Brownian particle with swimming speed $V_{0}$, subject to a gravitational force $\boldsymbol{F}$ and torque $\tau$ about the tracking point,

$$
\left(\begin{array}{c}
\dot{\boldsymbol{x}} \\
\dot{\theta}
\end{array}\right)=\left(\begin{array}{c}
V_{0} \cos \theta \\
V_{0} \sin \theta \\
0
\end{array}\right)+\boldsymbol{M}\left(\begin{array}{c}
\boldsymbol{F} \\
\tau
\end{array}\right)+\sqrt{2 k_{B} T} \boldsymbol{M}^{1 / 2} \boldsymbol{W}
$$

The $3 \times 3$ mobility matrix $\boldsymbol{M}$, calculated at the tracking point, couples the force and torque to the linear and angular velocities while $\boldsymbol{W} \in \mathbb{R}^{3}$ is a white noise vector that generates the Brownian motion. The force, $\boldsymbol{F}=-m g \boldsymbol{e}_{x} \sin \beta$, is proportional to $m$ the rod excess of mass over the displaced fluid, acceleration due to gravity $g$ and increases with the wall inclination $\beta$. The gravitational reorienting torque $\tau$ (normal to the $x y$-plane) has magnitude $r_{0} m g \sin \beta \cos \alpha \sin \theta$, where $r_{0}$ is the lever arm, i.e. the distance between the tracking point and the rod CoM.

Analyzing (4) is difficult because the rod translational and rotational dynamics are coupled. However, the orientation equation can be decoupled from the translation if the tracking point is chosen to be the center of rotation. The center of rotation is defined to be a pivot point about which an applied torque generates only rotation and not translation, whereas a net body force generates only translation and not rotation. [36 38] Such a pivot point is known to exist for two dimensional motion. 28] We denote this pivot point as the Center of Hydrodynamic stress $(\mathrm{CoH})$. Using the $\mathrm{CoH}$ as the tracking point the orientation equation simplifies to

$$
\frac{\mathrm{d} \theta}{\mathrm{d} t}=M_{\omega \tau} \tau+\sqrt{2 k_{B} T} M_{\omega \tau}^{1 / 2} W_{\theta} .
$$

For a rod in the bulk, far from any walls, the $\mathrm{CoH}$ is located at its geometric center. Near to a wall the $\mathrm{CoH}$ location may shift. For swimmers with a head-down tilt $(\alpha>0)$ the increased resistance near the front displaces the $\mathrm{CoH}$ headward from their geometric centers. 23] Therefore, the lever arm at which a body force exerts a gravitational torque can be decomposed into two contributions, $r_{0}=d_{\mathrm{CoM}}+d_{\mathrm{CoH}}$, i.e. distances measured from the rod's center to the $\mathrm{CoM}$ and $\mathrm{CoH}$, respectively; see Figs. 5 a, b. The larger the lever arm $r_{0}$, the larger the reorienting torque. This increased torque can dominate over the disorienting thermal fluctuations. Thus, once oriented upwards by the gravitational torque, a swimmer may move upwards gravitactically.

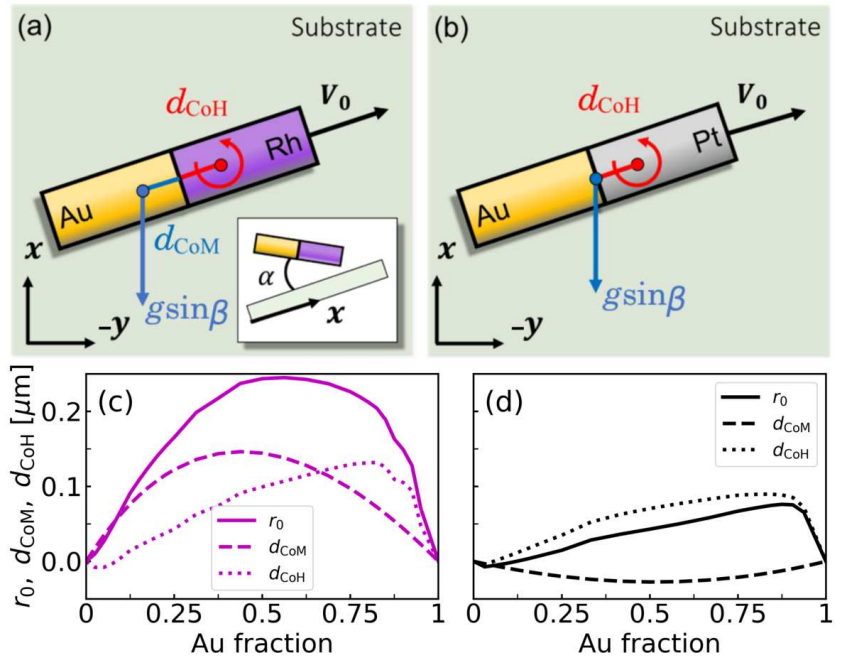

FIG. 5: Model for gravitaxis close to a wall. Body forces act on the rod's center of mass (CoM) whereas rotation occurs around the center of hydrodynamic stress $(\mathrm{CoH})$. (a) Tail-heavy rods experience a gravitational torque since CoM sits rearward and $\mathrm{CoH}$ headward. (b) Density-balanced rods experience a torque due only to the shifted $\mathrm{CoH}$ from the center. (c, d) Values for $d_{\mathrm{CoM}}$ and $d_{\mathrm{CoH}}$, the distances measured from the rod's center to the $\mathrm{CoM}$ and $\mathrm{CoH}$, respectively, from full hydrodynamic simulations, for (c) Au-Rh and (d) Au-Pt rods with varying gold lengths. Negative values of $d_{\mathrm{CoM}}$ indicate a CoM displaced headward.

A sizable level arm $r_{0}$ can be achieved using metals with density contrast (e.g. in the $\mathrm{Au}-\mathrm{Rh}$ case, the CoM sits rearwards). For the $\mathrm{Au}-\mathrm{Rh}$ rods, $d_{\mathrm{CoM}}$ is maximized for approximately symmetric rods, i.e. $L_{\mathrm{Au}} \approx L / 2$, see Figs. 55.

The $d_{\mathrm{CoH}}$ can be increased by moving the metal junction, and thus the location of the slip layer, headward. This fluid layer, which propels the rods, creates a pressure field that tilts the rods. 31] Such head-down tilt makes the leading portion of the rod closer to the solid wall than the trailing portion, see Fig. 1. The resulting resistance difference, higher near the head but lower at the tail, shifts the $\mathrm{CoH}$ headward and thus increases $d_{\mathrm{CoH}}$. Therefore, the location of the junction largely determines the position of the $\mathrm{CoH}$. Figs. 56, d illustrate the values (dotted curves) of $d_{\mathrm{CoH}}$, as functions of the position where two metals join, obtained with our full hydrodynamic model. Combining both contributions to the lever arm, $r_{0}$ (solid curves in Fig. 5s, d), the model predicts that the gravitactic effect for $\mathrm{Au}-\mathrm{Rh}$ rods will be maximized for length-symmetric swimmers while for Au-Pt rods will be maximized for rods with long gold segments. This is consistent with our experimental results shown in Fig. 4 some of these predictions are further validated next. 


\section{A. Quantifying the Lever Arm}

To test the coupled effects of gravity and hydrodynamic interactions with the wall, we examine the orientation of motile rods. The probability density function (PDF) of the angle $\theta$ can be calculated from Eq. (5). [19] The experiments measure the angle formed by the rod axis with the $x$-axis and do not distinguish the orientation $\theta$ from $\theta^{\prime}=\theta-180^{\circ}$. The PDF for the angle wrapped to $\left[-90^{\circ}, 90^{\circ}\right]$ is

$$
P(\theta)=\frac{e^{K \cos \theta}+e^{-K \cos \theta}}{2 \pi I_{0}(K)},
$$

where $I_{0}(K)$ is the modified Bessel function of order zero and $K=r_{0} m g \sin \beta \cos \alpha / k_{B} T$ is the ratio between the gravitational torque and the thermal energy, which randomizes the rod orientation. As shown in the supplement, upward swimming is possible when $K$ is larger than the ratio between the sedimentation velocity and the intrinsic swimming speed $V_{0}$. [23] The experimental results are depicted in Fig. 6a, b. The peaks at $\theta=0^{\circ}$ for both $\mathrm{Au}-\mathrm{Rh}$ and $\mathrm{Au}-\mathrm{Pt}$ rods are consistent with a lever arm $d_{\mathrm{CoH}}>0$ as predicted by the mechanical model.

From $P(\theta)$ we extracted the parameter $K$ that best fits the experimental results using Eq. (6); the values of $K$ versus the wall inclination are shown in Fig. 6r. As expected from the model, $K$ is proportional to $\sin \beta$. The results demonstrate that the overall torque is higher for $\mathrm{Au}-\mathrm{Rh}$ rods, for which $d_{\mathrm{CoM}}$ is significant. The values of the lever arm $r_{0}$ can be extracted by fitting the values of $K$ to the mechanical model prediction. For $\mathrm{Au}-\mathrm{Rh}$ rods the fit yields $r_{0}=0.19 \mu \mathrm{m}$, corresponding to $d_{\mathrm{CoH}}=$ $0.05 \mu \mathrm{m}$, which is ahead of the rod's midpoint because its $d_{\mathrm{CoM}}=0.14 \mu \mathrm{m}$. Here, hydrodynamic effects account for about $25 \%$ of the torque felt by $\mathrm{Au}-\mathrm{Rh}$ rods.

The Au-Pt rods were slightly head-heavy as platinum is denser than gold. In the cases of symmetric 1:1 Au:Pt and front-actuated 3:1 Au:Pt rods, $d_{\mathrm{CoM}}$ is -0.026 and $-0.02 \mu \mathrm{m}$, respectively. This contribution is insufficient to produce a bias in the rod orientation. The experimental data suggest a torque larger than the one created by the density mismatch. A fit of the experimental results reveals that the distance of the $\mathrm{CoH}$ to the geometric center is larger for asymmetric rods $\left(d_{\mathrm{CoH}}=0.14 \mu \mathrm{m}\right)$ than for the symmetric ones $\left(d_{\mathrm{CoH}}=0.076 \mu \mathrm{m}\right)$. This arm length difference generates the distinct sedimentation speeds seen in our two Au-Pt rod types.

These values of $K$ and $d_{\mathrm{CoH}}$ are larger than the ones obtained from Fig. 3 for immotile rods. For example, the values of $d_{\mathrm{CoH}}$ are $0.02,0.03$ and 0.03 for immotile $\mathrm{Au}-$ $\mathrm{Rh}, \mathrm{Au}-\mathrm{Pt}$ long gold and $\mathrm{Au}-\mathrm{Pt}$ symmetric respectively. Clearly, the rods' activity modify their orientations.
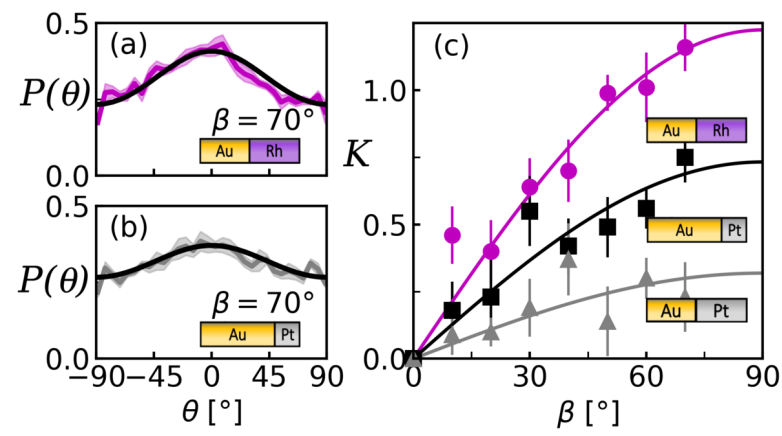

FIG. 6: Experiment vs. model. Experimental orientation distributions for motile (a) tail-heavy Au-Rh rods, and (b) asymmetric density-even $\mathrm{Au}-\mathrm{Pt}$ rods, show peaks at $\theta=0^{\circ}$. Fitting the data by Eq. 6) (solid curves) we obtained $K$. (c) The extracted $K$ values are plotted vs. tilt angle $\beta$. Values of $r_{0}$, further extracted by fitting $K$ to the theoretical formula, $K \sim r_{0} \sin \beta$ (solid lines), show gravitational torques act at lengths greater than $d_{\mathrm{CoM}}$ due to the shifted $\mathrm{CoH}$ in all 3 cases tail-heavy $(\bullet)$, density balanced asymmetric ( $\boldsymbol{\square})$ and density balanced symmetric rods $(\Delta)$.

\section{CONCLUSIONS}

Our results demonstrate gravitaxis using density unbalanced nanomotors fueled with $\mathrm{H}_{2} \mathrm{O}_{2}$. These "cliff climbers", which are about 15-20 times heavier than the surrounding fluid, move up steep walls. Interestingly, it is the gravitational pull that orients these tail-heavy rods and allows gravitaxis. Moreover, the emergent hydrodynamic effect when rods interact with the sloped walls [31, 39] enhances the effect. Such enhancement can be used to control the sedimentation speed of falling rods and promote gravitaxis.

The microswimmer behavior reveals that an imbalance in density of the two metals results in a reorienting gravitational torque, due to the shift of its center of mass. Additionally, the shift of the center of rotation reveals the importance of the hydrodynamic interactions. Both effects take place and contribute to successful gravitaxis. In the synthetic world, the mechanisms studied in this work can be employed to design efficient gravitactors as well as microswimmers which gravitaxis is triggered near walls.

\section{Acknowledgements}

This work was supported primarily by the MRSEC Program of the National Science Foundation under Award DMR-1420073, and also by NSF Grants DMSRTG-1646339, DMS-1463962 and DMS-1620331. JZ acknowledges partial support by Tamkeen under the NYU Abu Dhabi Research Institute grant CG002 and FBU acknowledges partial support from "la Caixa" Foundation (ID 100010434), fellowship LCF/BQ/PI20/11760014, and from the European Union's Horizon 2020 under the 
[1] A. M. Roberts, Journal of Experimental Biology 53, 687 (1970).

[2] J. O. Kessler, Nature 313, 218 (1985).

[3] A. M. Roberts and F. M. Deacon, Journal of Fluid Mechanics 452, 405 (2002).

[4] A. M. Roberts, Biological Bulletin 210, 78 (2006), ISSN 00063185.

[5] A. M. Roberts, Journal of Experimental Biology 213, 4158 (2010).

[6] P. R. Richter, D.-P. Hader, R. J. Goncalves, M. A. Marcoval, V. E. Villafane, and E. W. Helbling, Photochemistry and Photobiology 83, 810 (2007).

[7] Y. Mogami, J. Ishii, and S. A. Baba, The Biological Bulletin 201, 26 (2001).

[8] S. Childress, M. Levandowsky, and E. A. Spiegel, Journal of Fluid Mechanics 69, 591 (1975).

[9] K. Wolff, A. M. Hahn, and H. Stark, The European Physical Journal E 36, 43 (2013).

[10] W. Yan and J. F. Brady, Soft Matter 11, 6235 (2015).

[11] F. Rühle and H. Stark, arXiv preprint arXiv:2002.04323 (2020).

[12] F. Rühle, J. Blaschke, J.-T. Kuhr, and H. Stark, New Journal of Physics 20, 025003 (2018).

[13] S. Das, A. Garg, A. I. Campbell, J. Howse, A. Sen, D. Velegol, R. Golestanian, and S. J. Ebbens, Nat. Comm. 6, 8999 (2015).

[14] A. Petroff and A. Libchaber, Proceedings of the National Academy of Sciences 111, E537 (2014).

[15] A. P. Petroff, F. Tejera, and A. Libchaber, Journal of Statistical Physics 167, 763 (2017).

[16] D. P. Singh, W. E. Uspal, M. N. Popescu, L. G. Wilson, and P. Fischer, Advanced Functional Materials 28, 1706660 (2018).

[17] M. S. Rizvi, P. Peyla, A. Farutin, and C. Misbah, Phys. Rev. Fluids 5, 033101 (2020).

[18] J. Palacci, C. Cottin-Bizonne, C. Ybert, and L. Bocquet, Phys. Rev. Lett. 105, 088304 (2010).

[19] A. I. Campbell and S. J. Ebbens, Langmuir 29, 14066 (2013).

[20] B. ten Hagen, F. Kuemmel, R. Wittkowski, D. Takagi, H. Loewen, and C. Bechinger, Nature Communications 5, 4829 EP (2014).

[21] W. F. Paxton, P. T. Baker, T. R. Kline, Y. Wang, T. E. Mallouk, and A. Sen, J. Am. Chem. Soc. 128, 14881 (2006).

[22] M. J. Banholzer, L. Qin, J. E. Millstone, K. D. Osberg, and C. A. Mirkin, Nat. Prot. 4, 838 (2009).

[23] Supplemental Material URL to be inserted (????).

[24] Y. Wang, R. M. Hernandez, D. J. Bartlett, J. M. Bingham, T. R. Kline, A. Sen, and T. E. Mallouk, Langmuir 22, 10451 (2006).

[25] J. L. Moran and J. D. Posner, Physics of Fluids 26, 042001 (2014), https://doi.org/10.1063/1.4869328, URL https://doi.org/10.1063/1.4869328

[26] J. L. Moran and J. D. Posner, J. Fluid Mech. 680, 31 (2011).

[27] J. L. Moran and J. D. Posner, Ann. Rev. Fluid Mech. 49, 511 (2017).
[28] S. Delong, F. Balboa Usabiaga, and A. Donev, J. Chem. Phys. 143, 144107 (2015).

[29] F. Balboa Usabiaga, B. Kallemov, B. Delmotte, A. P. S. Bhalla, B. E. Griffith, and A. Donev, Comm. App. Math. Comp. Sci. 11, 217 (2016).

[30] B. Sprinkle, F. Balboa Usabiaga, N. A. Patankar, and A. Donev, J. Chem. Phys. 147, 244103 (2017).

[31] Q. Brosseau, F. Balboa Usabiaga, E. Lushi, Y. Wu, L. Ristroph, J. Zhang, M. Ward, and M. J. Shelley, Phys. Rev. Lett. 123, 178004 (2019).

[32] J. Rotne and S. Prager, J. Chem. Phys. 50, 4831 (1969).

[33] J. R. Blake, Math. Proc. Cam. Phil. Soc. 70, 303 (1971).

[34] J. W. Swan and J. F. Brady, Phys. Fluids 19, 113306 (2007).

[35] L. Ren, D. Zhou, Z. Mao, P. Xu, T. J. Huang, and T. E. Mallouk, ACS Nano 11, 10591 (2017).

[36] J. Happel and H. Brenner, Low Reynolds number hydrodynamics (Springer Netherlands, 1983).

[37] J. M. G. Bernal and J. G. De La Torre, Biopolymers 19, 751 (1980).

[38] R. N. Georgiev, S. O. Toscano, W. E. Uspal, B. Bet, S. Samin, R. van Roij, and H. B. Eral, Proceedings of the National Academy of Sciences 117, 21865 (2020), ISSN 0027-8424, https://www.pnas.org/content/117/36/21865.full.pdf, URL https://www.pnas. org/content/117/36/21865

[39] S. E. Spagnolie and E. Lauga, J. Fluid Mech. 700, 105 (2012). 


\title{
Metallic Microswimmers Driven up the Wall by Gravity
}

\author{
Quentin Brosseau $^{1}$, Florencio Balboa Usabiaga ${ }^{2}$, Enkeleida Lushi ${ }^{3}$, Yang $\mathrm{Wu}^{4}$, \\ Leif Ristroph $^{1}$, Michael D. Ward ${ }^{4}$, Michael J. Shelley ${ }^{1,2}$ and Jun Zhang ${ }^{1,5,6}$ \\ ${ }^{1}$ Applied Mathematics Laboratory, Courant Institute, New York University, NY NY 10012, USA, \\ 2 Flatiron Institute, Simons Foundation, NY NY 10010, USA \\ 3 Dept. of Math. Sciences, New Jersey Institute of Technology, Newark NJ 07102, USA \\ 4 Dept. of Chemistry, New York University, NY NY 10012, USA \\ 5 Dept. of Physics, New York University, NY NY 10003, USA \\ ${ }^{6}$ NYU-ECNU Institute of Physics, New York University Shanghai, Shanghai 200062, China
}

(Dated: July 29, 2021)

\section{EXPERIMENTAL DETAILS (SAMPLE PREPARATION)}

The nanorods are synthesized by a templating method on Anodic Aluminium Oxide (AAO) membranes (Whatman Anodisc $^{\mathrm{TM}} 47$ ) with a typical pore diameter of $0.3 \mu \mathrm{m}$. Prior to the electrodeposition, one side of the AAO membrane is sealed by thermo-evaporation of a $150 \mathrm{~nm}$ thick layer of silver (BAL-TEC MCS 010 Multi Control System).

The electrodeposition is made in three steps using a three-electrodes method:

- A layer of silver is deposited at $-1 \mathrm{~V}$ from an aqueous solution of silver cyanide $(0.0186 \mathrm{M}, \mathrm{AgCN}$, Thermo Fisher Scientific Inc.), potassium cyanide (0.1233 M, KCN, Thermo Fisher Scientific Inc.) and potassium pyrophosphate (0.0304 M, $\mathrm{K}_{4} \mathrm{P}_{2} \mathrm{O}_{7}$, Sigma-Aldrich, Co. LLC) to prevent leakages.

- A layer of gold is deposited at $-0.92 \mathrm{~V}$ from a commercial plating solution (OROTEMP 24 RTU Rack from TECHNIC INC).

- In the case of Au-Rh symmetric nanorods, a layer of rhodium is deposited at $-0.4 \mathrm{~V}$ from a commercial plating solution (Techni Rhodium RTU from TECHNIC INC). In this case, the deposition charges of gold $C_{\mathrm{Au}}$ and Rhodium $C_{\mathrm{Rh}}$ are $16 \mathrm{C}$ and $68 \mathrm{C}$, respectively.

In the case of $\mathrm{Au}-\mathrm{Pt}$ nanorods, a layer of platinum is deposited at $-0.4 \mathrm{~V}$ from an aqueous solution of ammonium hexachloroplatinate (IV) $\left(0.010 \mathrm{M},\left(\mathrm{NH}_{3}\right)_{2} \mathrm{PtCl}_{6}\right.$, Alfa Aesar) and sodium phosphate dibasic dihydrate $(0.020$ $\mathrm{M}, \mathrm{Na}_{2} \mathrm{HPO}_{4}$, Sigma-Aldrich, Co. LLC). The deposition charges of gold and platinum are $C_{\mathrm{Au}}=7.2 \mathrm{C}$ and platinum $C_{\mathrm{Pt}}=26 \mathrm{C}$ for symmetric rods and $C_{\mathrm{Au}}=24 \mathrm{C}$ and $C_{\mathrm{Pt}}=9 \mathrm{C}$ for long gold segment rods.

The silver layer is etched away in a solution of $\mathrm{HNO}_{3}(1 \mathrm{M})$, and the membrane is dissolved in a $\mathrm{NaOH}$ solution $(5$ $\mathrm{M})$. The resulting suspension with nanorods is purified through a repeated centrifugation/dilution process.

\section{A. Rods' parameters}

Table 1 shows the geometry of the rods, their diffusion coefficients $\left(D_{t}\right.$ and $\left.D_{r}\right)$ and their swimming speeds $\left(V_{0}\right)$ at different $\mathrm{H}_{2} \mathrm{O}_{2}$ concentration.

TABLE I: Table of geometrical and physical properties of symmetric Au-Rh nanorods and the two Au-Pt nanorod types with gold and platinum fractions $\left(l_{\mathrm{Au}}: l_{\mathrm{Pt}}\right)$.

\begin{tabular}{lcrrr}
\hline \hline Batch & $D_{t}\left[\mu \mathrm{m}^{2} / \mathrm{s}\right]$ & $D_{r}[1 / \mathrm{s}]$ & $V_{0}[\mu \mathrm{m} / \mathrm{s}]$ & $\mathrm{H}_{2} \mathrm{O}_{2} \%$ \\
\hline $\operatorname{Au}-\operatorname{Rh}(1: 1) L=2.5 \mu \mathrm{m}$ & 0.3 & 1.04 & 0 & 0 \\
$\operatorname{Au}-\operatorname{Rh}(1: 1) L=2.5 \mu \mathrm{m}$ & 0.3 & 1.04 & $2.7 \pm 0.3$ & 10 \\
$\operatorname{Au}-\operatorname{Rh}(1: 1) L=2.5 \mu \mathrm{m}$ & 0.3 & 1.04 & $4.5 \pm 0.5$ & 15 \\
$\operatorname{Au}-\operatorname{Rh}(1: 1) L=2.5 \mu \mathrm{m}$ & 0.3 & 1.04 & $8.0 \pm 1.0$ & 15 \\
$\operatorname{Au}-\operatorname{Pt}(1: 1) L=2 \mu \mathrm{m}$ & 0.25 & 0.6 & $6.1 \pm 0.8$ & 20 \\
$\operatorname{Au}-\operatorname{Pt}(3: 1) L=2 \mu \mathrm{m}$ & 0.25 & 0.6 & $6.5 \pm 0.7$ & 20 \\
\hline \hline
\end{tabular}




\section{CENTER OF HYDRODYNAMIC STRESS (COH)}

The choice of a tracking point to describe the orientation of a body cannot affect the dynamics of the system (of course!) but it affects the structure of the equations of motion. In systems with inertia, it is natural to choose the center of mass (CoM) as the tracking point because the translational and rotational contributions to the kinetic energy decouple. For particles immersed in a Stokes flow, where inertia does not play a role, other choices are more convenient. This issue was explored by Brenner in the 1960s [1] and later expanded and clarified by García Bernal and García de la Torre [2. We reproduce here their principal results for completeness.

Consider a rigid body immersed in a three dimensional Stokes flow. Its dynamics can be described by the linear and angular velocity about a tracking point 1 . The linear system that relates the force and torque $\left(\boldsymbol{F}_{1}\right.$ and $\left.\boldsymbol{\tau}_{1}\right)$ with the linear and angular velocities $\left(\boldsymbol{V}_{1}\right.$ and $\left.\boldsymbol{\omega}_{1}\right)$ is

$$
\left(\begin{array}{c}
\boldsymbol{V}_{1} \\
\boldsymbol{\omega}_{1}
\end{array}\right)=\left(\begin{array}{ll}
\boldsymbol{M}_{V F, 1} & \boldsymbol{M}_{\omega F, 1}^{T} \\
\boldsymbol{M}_{\omega F, 1} & \boldsymbol{M}_{\omega \tau, 1}
\end{array}\right)\left(\begin{array}{c}
\boldsymbol{F}_{1} \\
\boldsymbol{\tau}_{1}
\end{array}\right)
$$

where the $3 \times 3$ mobility components $\boldsymbol{M}_{V F, 1}$ etc. depend on the tracking point chosen to describe the motion as indicated by the subindex 1. The force, torque and velocities defined at a second tracking point are

$$
\begin{gathered}
\boldsymbol{F}_{2}=\boldsymbol{F}_{1}, \quad \boldsymbol{\tau}_{2}=\boldsymbol{\tau}_{1}-\boldsymbol{r} \times \boldsymbol{F}_{1}, \\
\boldsymbol{V}_{2}=\boldsymbol{V}_{1}+\boldsymbol{\omega}_{1} \times \boldsymbol{r}, \quad \boldsymbol{\omega}_{2}=\boldsymbol{\omega}_{1},
\end{gathered}
$$

where $\boldsymbol{r}$ is the vector that goes from the first to the second tracking point, see Fig. 1 1 left. We can use (1)-(3) to show that the mobility components transform between tracking points like [2]

$$
\begin{aligned}
& \boldsymbol{M}_{\omega \tau, 2}=M_{\omega \tau, 1}, \\
& M_{\omega F, 2}=M_{\omega F, 1}+M_{\omega \tau, 1} \times \boldsymbol{r} \\
& M_{V F, 2}=M_{V F, 1}-\boldsymbol{r} \times\left(M_{\omega \tau, 1} \times \boldsymbol{r}\right)+M_{\omega F, 1}^{T} \times \boldsymbol{r}-\boldsymbol{r} \times \boldsymbol{M}_{\omega F, 1},
\end{aligned}
$$

where the cross product between a $3 \times 3$ matrix and a vector is defined, using the Levi-Civita symbol, as $(\boldsymbol{M} \times \boldsymbol{r})_{i j}=$ $M_{i k} \epsilon_{j k l} r_{l}$ and $\boldsymbol{r} \times \boldsymbol{M}=-\boldsymbol{M} \times \boldsymbol{r}$.

For any body shape there is a special tracking point where the coupling matrix $\boldsymbol{M}_{\omega F}$ is symmetric. This point is called in the literature the Center of Mobility [2]. The location of the center of mobility with respect to an arbitrary tracking point can be found by solving for $i \neq j$ the linear system 3 ]

$$
\left(\epsilon_{i k l}\left(\boldsymbol{M}_{\omega \tau}\right)_{j k}-\epsilon_{j k l}\left(\boldsymbol{M}_{\omega \tau}\right)_{i k}\right) r_{l}=\left(\boldsymbol{M}_{\omega F}\right)_{i j}-\left(\boldsymbol{M}_{\omega F}\right)_{j i}
$$

where the mobility components are calculated at the original tracking point. For bodies of enough symmetry (e.g. axisymmetric bodies), the coupling matrix $\boldsymbol{M}_{\omega F}$ vanishes at the center of mobility. In such cases, the center of mobility is also called the Center of Hydrodynamic stress $(\mathrm{CoH})$. Therefore the $\mathrm{CoH}$ can be found from (7) if it exists.

For two dimensional systems (or three dimensional particles constrained to move in the $x y$ plane), the CoH always exists and it corresponds to the point where a torque applied out of the plane does not generate translations. We can compute its location respect an arbitrary tracking point with [3]

$$
\boldsymbol{r}=\left(\frac{M_{\omega_{z} F_{y}}}{M_{\omega_{z} \tau_{z}}}, \frac{M_{\omega_{z} F_{x}}}{M_{\omega_{z} \tau_{z}}}\right)
$$

As discussed in the main text, we use this tracking point to uncouple the rotational equation of motion from translations.

The figure 1 right shows that if the hydrodynamic interactions with the wall are neglected the density mismatch is not enough to explain the orientation bias of the rods observed in the experiments.

\section{EFFECT OF THE SWIMMING SPEED}

In this section we estimate the critical swimming speed that allows upward movement (i.e. $\left.\left\langle V_{x}\right\rangle>0\right)$. When the reorienting torque is very large (i.e. $K \gg 1$ ) the rods are aligned with the $x$-axis and the critical swimming speed coincides with the speed of sedimentation along the wall $V_{0 c}=\mu_{\|} m g \sin \beta$, here $\mu_{\|}$is the tangential mobility of the 

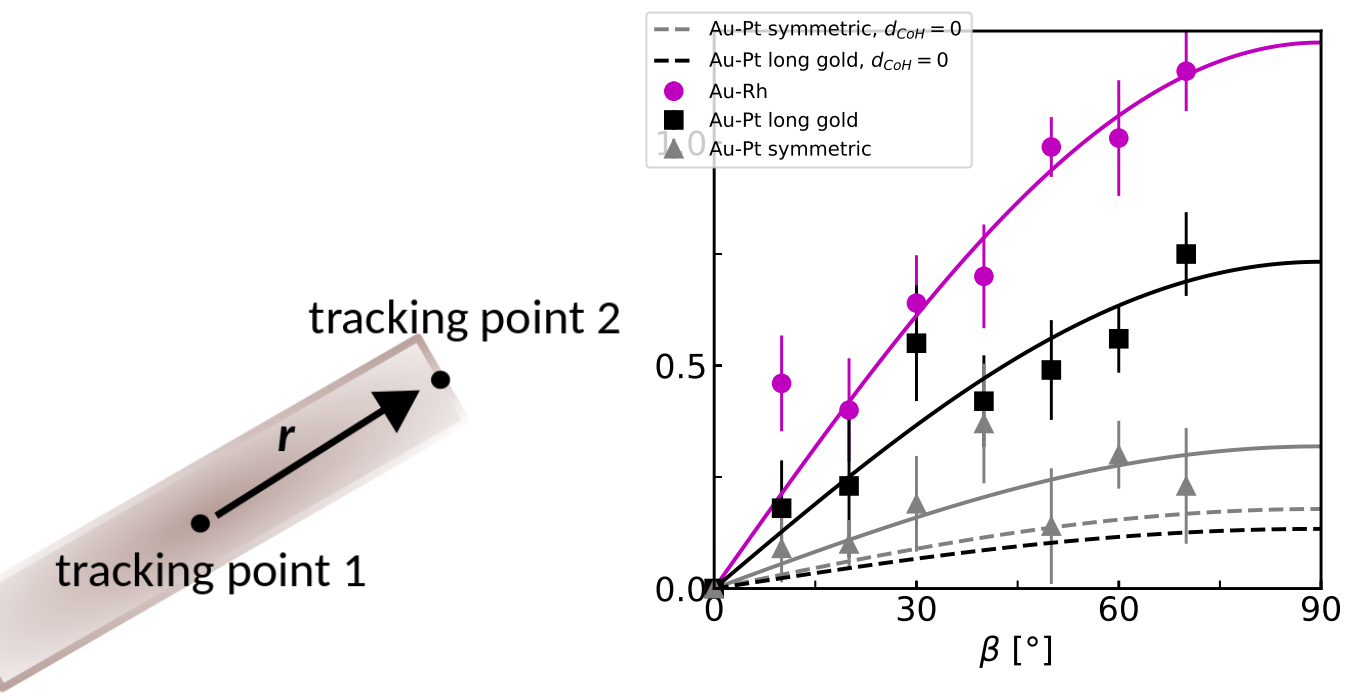

FIG. 1: (Left) Sketch of a rigid body with two tracking points. (Right) Reproduction of the figure 6c from the main text, $K$ vs. $\beta$, for experiments (symbols) and theoretical fits (continuum lines). We include the theoretical prediction assuming $d_{\mathrm{CoH}}=0$ (dashed lines) which reveals the important effect of a finite $d_{\mathrm{CoH}}$ to fit the experimental results.

rod. For weak reorienting torques, $K \lesssim 1$, like in our experiments the critical swimming speed will be larger as the rods are not always aligned with the $x$-axis. We use our mechanical model to estimate $V_{0 c}$ in this regime. The linear velocity of the Center of Hydrodynamic stress ( $\mathrm{CoH})$ is (from eq. [1] in the main text)

$$
\boldsymbol{V}=\left(\begin{array}{c}
V_{0} \cos \theta \\
V_{0} \sin \theta
\end{array}\right)+\boldsymbol{M}_{V F} \boldsymbol{F}+[\text { noise terms }]
$$

note that the torque does not appear explicitly because we use the $\mathrm{CoH}$ as the tracking point. The mobility depends on the angle $\theta$ between the rod and the $x$-axis

$$
\boldsymbol{M}_{V F}=\left(\begin{array}{cc}
\cos \theta & -\sin \theta \\
\sin \theta & \cos \theta
\end{array}\right)\left(\begin{array}{cc}
\mu_{\|} & 0 \\
0 & \mu_{\perp}
\end{array}\right)\left(\begin{array}{cc}
\cos \theta & -\sin \theta \\
\sin \theta & \cos \theta
\end{array}\right)^{T}
$$

where $\mu_{\|}$and $\mu_{\perp}$ are the parallel and perpendicular mobilities (for a slender body $\mu_{\|}=2 \mu_{\perp}$ ). Therefore, the velocity along the $x$-axis is

$$
V_{x}=V_{0} \cos \theta+\mu_{\perp} F_{x}+\left(\mu_{\|}-\mu_{\perp}\right) F_{x} \cos ^{2} \theta+[\text { noise terms }],
$$

and after integrating over orientations we get

$$
\left\langle V_{x}\right\rangle=\frac{1}{2 \pi} \int_{-\pi}^{\pi} V_{x} P(\theta) \mathrm{d} \theta=V_{0} \frac{I_{1}(K)}{I_{0}(K)}+\frac{1}{2}\left[\left(\mu_{\|}+\mu_{\perp}\right)+\left(\mu_{\|}-\mu_{\perp}\right) \frac{I_{2}(K)}{I_{0}(K)}\right] F_{x},
$$

where $I_{n}(x)$ are modified Bessel functions of the first kind and we used the angle distribution, $P(\theta)=$ $\exp (K \cos \theta) /\left(2 \pi I_{0}(K)\right)$, obtained from the eq. [2] in the main text. To first order in $K$

$$
\left\langle V_{x}\right\rangle=\frac{V_{0} K}{2}+\frac{\mu_{\|}+\mu_{\perp}}{2} F_{x} .
$$

Upward swimming $\left(\left\langle V_{x}\right\rangle>0\right)$ is possible when $K>-\left(\mu_{\|}+\mu_{\perp}\right) F_{x} / V_{0}$, i.e. when $K$ is larger than the ratio between the sedimentation velocity and the intrinsic swimming speed. After substituting the values of the gravitational force, $F_{x}=-m g \sin \beta$, and $K=r_{0} m g \sin \beta \cos \alpha / k_{B} T$, we obtain the critical swimming speed for weak reorienting torques

$$
V_{0 c}=\frac{\left(\mu_{\|}+\mu_{\perp}\right) k_{B} T}{r_{0} \cos \alpha} .
$$




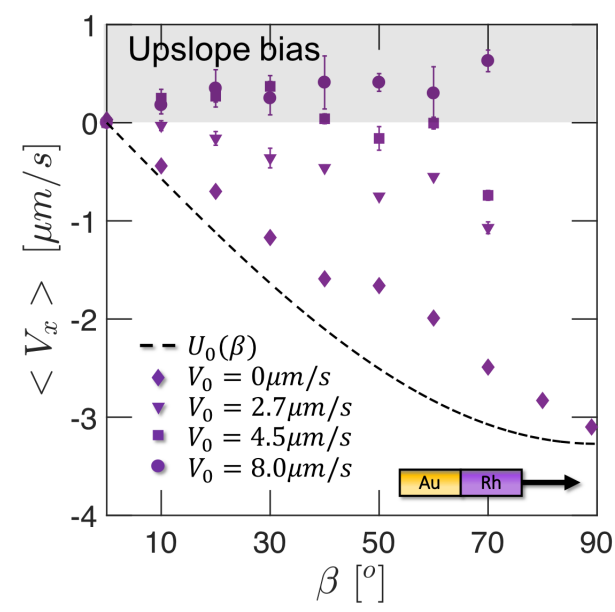

FIG. 2: Experimental results (symbols) of gravitaxis of bottom-heavy rods for several swimming speeds controlled with the $\mathrm{H}_{2} \mathrm{O}_{2}$ concentration. The dashed line represents the sedimentation velocity estimation $U_{0}(\beta)=-\mu_{\|} m g \sin \beta$.

Interestingly, in this regime the critical swimming speed is independent of the particle mass and the inclination of the wall, as long as $m g \sin \beta>0$, because the gravitational pulling force contributes both to reorient the particle upwards and to pull it downwards. The length of the lever arm, $r_{0}$, is critical.

Using the mobility approximation of a cylinder in bulk $\left(\mu_{\|}=(\log (L / r)-0.72) /(2 \pi \eta L)\right.$ and $\left.\mu_{\perp}=\mu_{\|} / 2[1]\right)$ we estimate a critical swimming speed of around $4 \mu \mathrm{m} / \mathrm{s}$ for our Au-Rh particles. Indeed, Fig. 2 shows that slow rods fall for all wall inclinations. Faster rods with $V_{0}=4.5 \mu \mathrm{m} / \mathrm{s}$ show upslope motion (shaded area) for moderate values of $\beta$. For higher swimming speeds, upslope motion is visible for all inclinations.

\section{ADDITIONAL NUMERICAL RESULTS}

We provide here some additional results obtained from numerical simulations to support our claims. First, we show the tilt angle $\alpha$ of rods towards the wall in the left panel of figure 3 . Rods with long gold segments tilt more. This is consistent with our previous investigation about the dynamic of phoretic swimmers in shear flows 4. Note that the tilt angle is controlled by the location of the active slip along the rod and that the density difference between Au-Rh and Au-Pt plays a minimal role. We show the computed distance between the $\mathrm{CoH}$ and the rod's center in Fig. 3 right. The physical interpretation of these results is that the higher drag near the front of the rod displaces the center of rotation forward. Note that in the limit where a rod is pinned to the wall the anchor point will act as the center of rotation.

In simulations with Brownian noise we are able to measure the average velocity along the wall $\left\langle V_{x}\right\rangle$ and the orientation bias measured by $K$ just like in the experiments. Brownian motion can be included into our model by adding a stochastic contribution, $\boldsymbol{u}_{i}^{\text {stoch }}=\sqrt{2 k_{B} T / \Delta t}\left(\boldsymbol{M}^{1 / 2} \boldsymbol{W}\right)_{i}$, to the right hand side of the slip condition, Eq. 1 in the main text, and using an stochastic integrator to update the rod position and orientation 3 , 5.

In the top panels of Fig. 44 we show the average velocity along the $x$-axis versus the wall inclination for Au-Pt and $\mathrm{Au}-\mathrm{Rh}$ rods. In the bottom panels of Fig. 4. we show $K$ versus the wall inclination $\beta$. The Au-Rh rods swim upwards for all inclinations and all swimmer types. Meanwhile, Au-Pt rods with short-gold segement and symmetric swimmers fall although their orientations show that they point upwards most of the time. Au-Pt rods with long-gold segment show a weak upward swimming bias. All these results agree qualitatively with the experiments although the numerical swimmers are better gravitactors. We note that in the simulations we model the active slip instead of solving the complicated electrochemical problem that ultimately creates the active flows. Moreover, we ignore if there are electrostatic forces between the rods and the wall which could affect the results. For these reasons we do not expect a perfect agreement between the simulations and the experimental results. The great contribution from the simulations is to show, in a perfect controlled system, the same general behavior as in the experiments. 

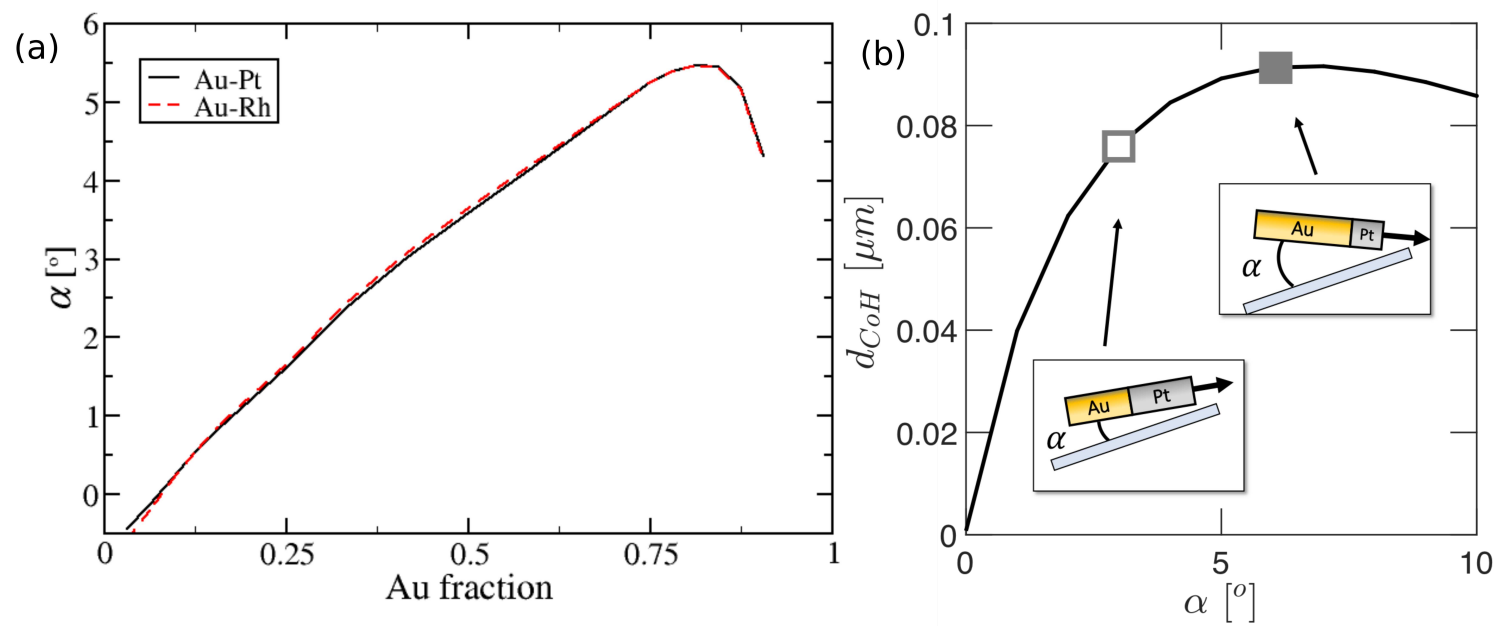

FIG. 3: Results from deterministic simulations for $L=2 \mu \mathrm{m}$ long rods. (a) tilt angle $\alpha$ with the wall for rods with different gold fractions. (b) Distance between the $\mathrm{CoH}$ and the rod's geometric center assuming that the rod's head is at a height $h=0.2 \mu \mathrm{m}$ from the wall.
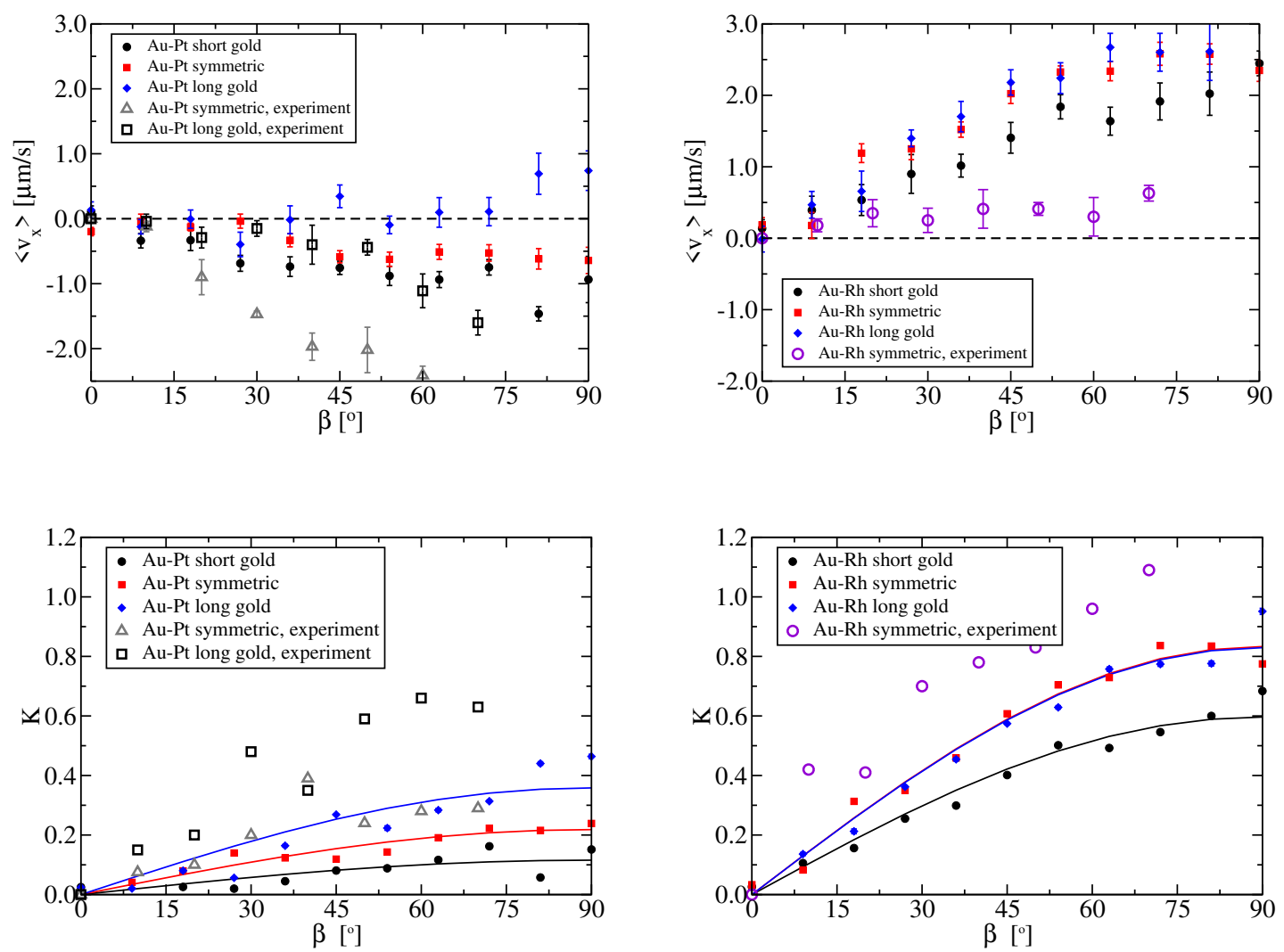

FIG. 4: Comparison between experimental results (open symbols) and Brownian simulations (full symbols) of $2 \mu \mathrm{m}$ long rods swimming near a wall. Top panels: mean upward velocity versus wall inclination for Au-Pt (Left) and Au-Rh rods (Right). Bottom panels: $K$ parameter versus wall inclination for $\mathrm{Au}-\mathrm{Pt}$ (Left) and $\mathrm{Au}-\mathrm{Rh}$ rods (Right). The continuum lines are fit of the numerical results to the theoretical formula. 
[1] J. Happel and H. Brenner, Low Reynolds number hydrodynamics (Springer Netherlands, 1983).

[2] J. M. G. Bernal and J. G. De La Torre, Biopolymers 19, 751 (1980).

[3] S. Delong, F. Balboa Usabiaga, and A. Donev, J. Chem. Phys. 143, 144107 (2015).

[4] Q. Brosseau, F. Balboa Usabiaga, E. Lushi, Y. Wu, L. Ristroph, J. Zhang, M. Ward, and M. J. Shelley, Phys. Rev. Lett. 123, 178004 (2019).

[5] B. Sprinkle, F. Balboa Usabiaga, N. A. Patankar, and A. Donev, J. Chem. Phys. 147, 244103 (2017). 